\title{
ASSESSMENT OF THE TECHNICAL LEVEL OF MAGNETIC FISHING TOOLS
}

\author{
Taras ROMANYSHYN, Liubomyr ROMANYSHYN \\ Ivano-Frankivsk National Technical University of Oil and Gas \\ Michal BEMBENEK \\ AGH University of Science and Technology \\ Ihor MOKHNII \\ Branch "UGV-SERVICE" JSC "Ukrgasvydobuvannya"
}

\begin{abstract}
:
A variety of magnetic fishing tools poses the task of the optimal choice of tool for eliminating accidents during the construction, operation and repair of wells. Existing criteria for assessing the quality of fishing magnets are characterized by the complexity of the determination and the ambiguity of the results. Therefore, the aim of research is development of a new approach to determining the technical level of fishing tools of various types and designs. A complex criterion has been developed that allows to evaluate the technical level of magnetic systems by correlating the actual and theoretical values of the total and specific lifting forces. Also it has been carried out a qualimetric analysis of magnetic tools, which are currently offered by world manufacturers. As a result, mathematical models are found that describe the average and modern world level of devices with specific lifting force. Technical decisions are proposed, the implementation of which in the design of magnetic systems of fishing tools will allow to achieve high values of lifting force. Application of the proposed complex criterion along with the results of qualimetric analysis will make it possible to objectively assess the technical level of magnetic fishing tools both at the design stage and during serial production.
\end{abstract}

Key words: fishing tool, lifting force, magnetic system, permanent magnet, technical level

\section{INTRODUCTION}

Accidents happening during the construction and operation of wells are characterized by a wide variety, as a result, metal debris remain on the bore-hole bottom $[1,2]$. In addition, a lot of metal cuttings remain in the wells after the milling operations as well as sidetracking [3]. Haughton and Connell [4] suggested that circulation of drilling mud do not transport larger metal debris to the surface. These cuttings accompany the drilling process and may cause the failure of the rock destruction tool. It should be noted that the volume of well repair work is growing annually [5], during which the tool and metal objects often fall into the well. Statistics indicate that fishing operations approximately accounted for $25 \%$ of drilling costs worldwide [6].

To retrieve emergency objects from the well without their preliminary destruction, fishing tools of mechanical, hydraulic and magnetic action are used [7, 8]. It is established $[9,10]$ that tools based on permanent magnets are the most effective of all types of fishing tools. They make it possible to retrieve small ferromagnetic objects of various shapes, sizes, masses and bottom-hole positions. The effectiveness of accident elimination is primarily determined by the right choice of fishing tool. Using the wrong tool leads to an increase in the time and money spent on emergency recovery work in the wells. In some cases, this can lead to a complication of the accident or even liquidation of the well.

Magnetic fishing tools, which are currently manufactured in the world, differ in the way of creation of a magnetic field, the design of the magnetic system and the material of permanent magnets. A wide variety of magnetic devices of the same size leads to the problem of rational selection of tools for efficient fishing operations. So, the aim of research is development of a new approach to assessing the technical level of magnetic fishing tools of various types and designs.

\section{LITERATURE REVIEW}

In the conditions of the modern development of equipment and technology for repair work, the requirements for magnetic fishing tools are significantly increasing. 
Their quality and technical level is assessed by a set of characteristics. The efficiency of the devices during the removal of metal objects from the wells directly depends on the power characteristics of their magnetic systems. The main parameter of fishing magnets is the lifting force $F$, which characterizes the value of magnetic energy in the working gap and is determined by the wellknown Maxwell formula:

$$
F=\frac{B^{2} S_{S}}{2 \mu_{0}}
$$

where:

$B$ - magnetic flux density in the working gap,

$S_{S}$ - area of the working surface of the magnetic cores, $\mu_{0}=4 \pi \cdot 10^{-7}-$ magnetic constant.

According to formula (1), the maximum lifting force can be obtained by saturating the magnetic cores to the maximum possible values of magnetic flux density and increasing the area of the poles, concentrating and directing the magnetic flux into the fished object. Magnetic cores should occupy the largest possible area of the fishing tool's working surface. Therefore, the constructive perfection of the magnetic system is determined by the utilization of the pole area $k_{p}[11,12]$ :

$$
k_{p}=\frac{s_{s}}{s_{t}}
$$

where:

$S_{t}$ - total area of the working surface of the magnetic system.

For general-purpose magnetic power systems, $k_{p}=0.3-$ 0.8. Considering that part of the working area is occupied by permanent magnets and circulation holes, for magnetic systems of fishing tools $k_{p}$ is approaching 0.8 . Objects of various shapes and masses remain on the bottom of the well, so their contact area with the magnetic cores is much smaller than the area of the system. Therefore, the relevant parameter for fishing tools is also the specific lifting force $F_{s}$ :

$$
F_{S}=\frac{F}{S_{s}}
$$

Along with the lifting force $F$, an important force parameter is the attractive force $F_{a t}$, which characterizes the degree of force acting on an object at a distance $\delta$ from the system [13]. It is possible to assess $F_{a t}$ with traction characteristics:

$$
F_{a t}=f(\delta)
$$

While fishing magnets fish ferromagnetic objects, the working gap $\delta$ is constantly changing. The work done to attract the object to the poles determines the energy of the magnetic system with a certain gap [13]:

$$
W_{m s}=\int_{0}^{\delta} F(\delta) d \delta
$$

where:

$F(\delta)$ - the force created by the magnetic system in the working gap.
The energy of the magnetic system $W_{m s}$ is a general power characteristic that takes into account both the maximum attractive force and the steepness of the traction characteristic.

The quality of the magnetic system is also determined by the scattering coefficient $k_{s c}$ [12], which characterizes the magnitude of the magnetic flux loss:

$$
k_{s c}=\frac{\Phi_{0}}{\Phi_{w}}
$$

where:

$\Phi_{\mathrm{o}}$ - full magnetic flux of the permanent magnets,

$\Phi_{w}$ - working magnetic flux on the surfaces of the magnetic cores.

In addition, when assessing the effectiveness of magnetic fishing tools, it is also necessary to take into account the stability of the parameters of the magnetic system, mechanical strength, dimensions, weight, and the cost of the device. However, these parameters do not significantly affect the rational choice of a tool for fishing operation. Indeed, the mechanical strength and dimensions of the device are limited by design requirements and difficult operating conditions of the magnetic tool in wells for various purposes. And such factors as the utilization of the area of the poles, the scattering coefficient, and the stability of the parameters are determined by the design of the magnetic system and the materials of the permanent magnets.

Thus, the main parameters and criteria for assessing the effectiveness of magnetic devices include the maximum and specific lifting force, traction characteristic, as well as the energy of the magnetic system. A more detailed analysis of these characteristics makes it possible to establish certain patterns. So, to fish ferromagnetic objects, it is necessary to create maximum lifting force. On the bore-hole bottoms there is a gap between the fished objects and the magnetic system. Therefore, to attract ferromagnetic debris, it is necessary to create a sufficient magnetic field. That is, the fishing tool must have a flat traction characteristic. It is possible to increase the area of the force of a magnetic field on objects by increasing the length of the permanent magnet. However, due to the limited external diameter of the system, this leads to a decrease in the area of the working surface of the poles and, as a result, a decrease in the total lifting force. Thus, a technical contradiction arises - an increase in the lifting force inevitably leads to a decrease in the attractive force in the working gap, i.e., to obtain an abrupt traction characteristic.

This example clearly shows the close relationship of the parameters with each other. Therefore, for the correct assessment of the technical level of the magnetic tool, it is necessary to take into account the whole complex of criteria. It is worth noting that identifying some of them is a complex and cumbersome task. 
DEVELOPMENT OF COMPLEX CRITERION FOR THE ASSESSMENT OF THE TECHNICAL LEVEL OF MAGNETIC FISHING TOOLS

Based on the analysis of the main parameters and criteria for assessing the effectiveness of fishing tools, their complex dependence on the design features of magnetic systems, materials of permanent magnets and magnetic cores, as well as operating conditions, is established. Therefore, to assess the operational parameters of the systems, a complex criterion $k_{c}$ is proposed. Using the ratios of the achieved and limit values of the total and specific lifting force, the criterion $k_{c}$ allows to objectively assess the efficiency of magnetic devices:

$$
k_{c}=k_{t} \cdot k_{s}
$$

where:

$k_{t}$ - coefficient of total lifting force,

$k_{s}$ - coefficient of specific lifting force.

The coefficient of total lifting force is determined as follows:

$$
k_{t}=\frac{F_{c}}{F_{t h}}
$$

where:

$F_{c}$ - conditional lifting force. $F_{c}$ is determined during testing, provided that the working surface of the magnetic system is completely contacted by a plate made of steel 1010.

$F_{t h}$ - theoretically achievable lifting force. The value of the theoretical lifting force is determined provided that the magnetic cores fill the entire area of the magnetic system. Specific lifting force coefficient:

$$
k_{s}=\frac{F_{s}}{F_{s t h}}
$$

where:

$F_{\text {sth }}$ - the theoretically achievable specific lifting force, $F_{\text {sth }}=1.82 \mathrm{~N} / \mathrm{mm}^{2}[13]$.

Theoretical values of the total $F_{t h}$ and specific $F_{s t h}$ lifting force are determined by the Maxwell formula (1).

Then, expressing $F_{s}$ in terms of (3), the complex criterion $k_{\mathrm{c}}$ will take the form:

$$
k_{c}=\frac{4 \mu_{0}^{2} \cdot F_{c}^{2}}{B_{m}{ }^{4} \cdot S_{s} \cdot S_{t}}
$$

where:

$B_{m}$ - maximum magnetic flux density for soft magnetic material.

\section{METHODOLOGY OF RESEARCH}

In order to assess the technical level of the currently known serial models of magnetic fishing tools, their qualimetric analysis is also carried out. For this purpose, the passport parameters of fishing magnets of 185 models manufactured by 15 engineering companies in six countries of the world have been collected. It is worth noting that some manufacturers currently do not exist, but their products continue to be used, which gives reason to enter it into the total data.
When conducting qualimetric analysis, methods of mathematical statistics are used, including the apparatus of regression analysis. For the study, the following parameters are selected: outer diameter $D$, specific lifting force $F_{s}$, mass $m$.

Obviously, the lifting force increases with an increase in the diameter of the device. This is due to the large area of the poles of the magnetic cores, and not implemented design concepts or a reasonable choice of materials. Therefore, in order to achieve the generality of the studied parameters of devices of various diameters (the ability to consider the parameters of the entire set of models as a single sample), the above parameters are replaced by their specific equivalents:

- specific lifting force $4 F / \pi D^{2}, \mathrm{~N} / \mathrm{mm}^{2}$;

- specific material consumption $m / F, \mathrm{~kg} / \mathrm{N}$.

Unfortunately, information on the mass of fishing tools is very limited: no more than a third of manufacturers report its value. Therefore, it is impossible to obtain a representative sample, which forced us to abandon the study of the parameter of specific material consumption.

To study the lifting force from the values of specific parameters $4 F / \pi D^{2}$ and passport parameters $D$, a two-dimensional sample is formed and tested for homogeneity. By methods of regression analysis, a two-dimensional array is approximated by monotonic continuous functions $4 F / \pi D^{2}=$ $f(D)$. The result is a mathematical model that reflects the average technical level of the studied models of magnetic fishing tools.

Also important is the task of determining the parameters of the magnetic tools corresponding to the modern world level. For this, those from which the relative deviations of the specific lifting force calculated by the equation of the average level are positive values are selected from the general set of models. The resulting array is subjected to regression analysis, similar to a full array of data. The obtained empirical equation describes the current world level of magnetic fishing tools with a specific lifting coefficient.

\section{RESEARCH RESULTS OF THE TECHNICAL LEVEL OF MA- GNETIC FISHING TOOLS}

According to the results of the qualimetric analysis, equation (11) and (12) is obtained, which are mathematical models of the average and modern world level for the studied models of magnetic fishing tools:

$$
\begin{aligned}
\frac{4 F}{\pi D^{2}} & =0.0911 \cdot D^{0.176} \\
\frac{4 F}{\pi D^{2}} & =0.4796 \cdot D^{-0.059}
\end{aligned}
$$

It is worth noting that power dependence is chosen as an approximating function, devoid of local extrema in the region of specifying the argument, and with minimal standard deviations describes the empirical dependencies under study. The function graphs constructed according to equations (11) and (12) are shown in Fig. 1. 


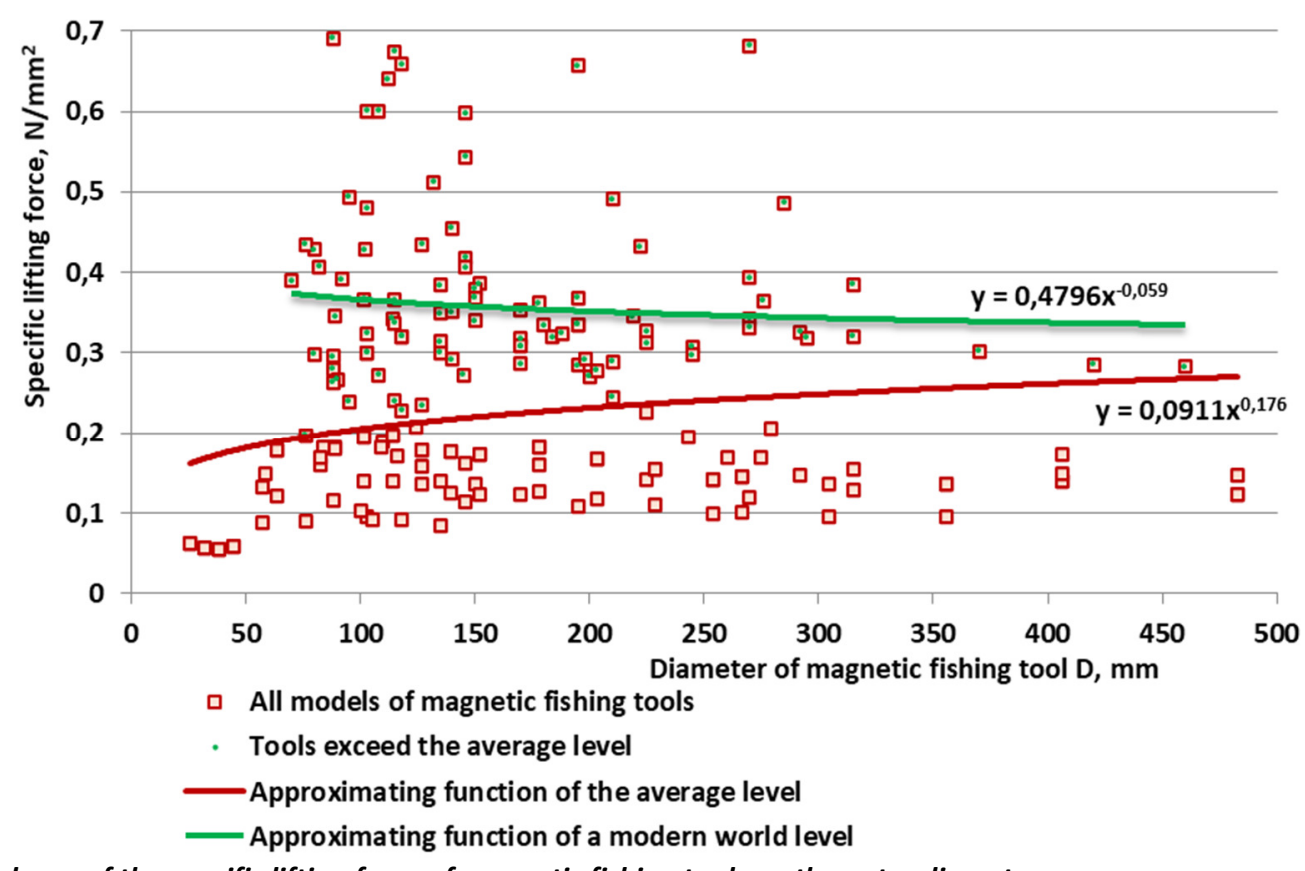

Fig. 1 The dependence of the specific lifting force of magnetic fishing tools on the outer diameter

As it is possible to see, the models of magnetic tools located below the graph of the average level do not correspond to the average technical level. The location of the point between the two functions indicates that the fishing magnet exceeds the average technical level, but does not reach the modern world level. And, accordingly, the presence of a point above the function of the modern world level indicates the perfection and competitiveness of such models on the world market.

Assessment of the technical level of magnetic fishing tools according to the proposed complex criterion $k_{c}$ is given for devices $[14,15]$. For this, its components are found - specific $k_{s}$ and total $k_{t}$ force coefficients. In these fishing tools, the average values of the specific lifting force are close to the theoretically achievable value of $1.82 \mathrm{~N} / \mathrm{mm}^{2}$. Therefore, the coefficient $k_{s}$ calculated according to (9) is in the range 0.90-0.95 (Table 1 ).

Table 1

Design parameters of magnetic systems

\begin{tabular}{|c|c|c|c|c|c|c|c|}
\hline 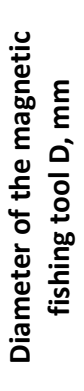 & 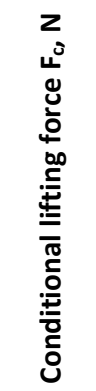 & 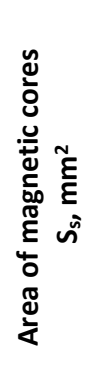 & 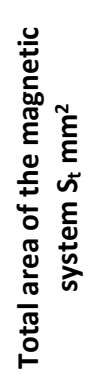 & 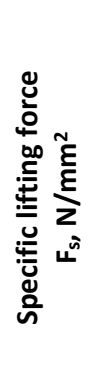 & 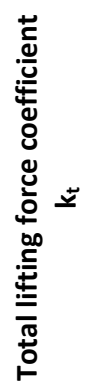 & 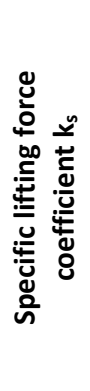 & 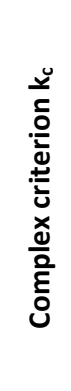 \\
\hline 90 & 5000 & 4183 & 3048 & 1.64 & 0.66 & 0.90 & 0.59 \\
\hline 115 & 8200 & 7085 & 4916 & 1.67 & 0.63 & 0.92 & 0.58 \\
\hline 136 & 11000 & 9847 & 6629 & 1.66 & 0.61 & 0.91 & 0.56 \\
\hline 195 & 19400 & 17662 & 11242 & 1.73 & 0.60 & 0.95 & 0.57 \\
\hline 270 & 42100 & 39740 & 25160 & 1.67 & 0.58 & 0.92 & 0.53 \\
\hline
\end{tabular}

The theoretical values of the total force $F$ are calculated provided that the magnetic cores fill the entire area of the magnetic system. Since circulation holes and permanent magnets occupy part of the working surface area of the system, according to (8), relatively small values of the total lifting coefficient $k_{t}$ are obtained. As a result, the complex criterion $k_{c}$ is from 0.53 to 0.59 , depending on the diameter of the fishing tool.

\section{DISCUSSION}

The obtained mathematical models (11), (12) allow to assess the technical level of magnetic fishing tools and solve the problem of the optimal choice of a particular model. So, these dependencies can be used at the stage of designing tools based on permanent magnets, namely to determine the parameters corresponding to the modern world level. It is also possible to assess the competitiveness of fishing magnets that are already mass-produced at a specialized engineering company. In addition, with their help, it is possible to identify potentially promising models for the acquisition.

The technical level of magnetic fishing tools can be faster, but with less accuracy determined graphically using Fig. 1. To do this, it is necessary to calculate the specific parameter of the lifting force and reflect the point corresponding to the model under study. This will allow for a qualitative assessment of the tool with a specific lifting force coefficient and assign it to one of the categories:

1) the model does not reach the average technical level;

2) the model corresponds to the average level, but does not reach the modern world level;

3) the model exceeds both the average and the modern world technical level. 
It is worth noting that the rapid development of science and technology contributes to the intensive updating of the range of oil and gas equipment and tools, including magnetic fishing tools. The results can be updated by adding new models and removing obsolete ones with the subsequent finding of approximating functions.

The search for technical decisions the implementation of which in the design of fishing magnets will provide them with a high technical level deserves special attention. Analysis of graphical dependencies (Fig. 1) shows that tools with alnico or ceramic permanent magnets are below the functions of the modern world level. Devices with highly coercive neodymium and samarium-cobalt permanent magnets are world-class. It should be noted that these materials are much more expensive than ceramic or alnico permanent magnets [16], which significantly increases the cost of the fishing tool.

However, as a result of a more detailed analysis, it is possible to conclude that fishing tools with rare-earth magnets have a different technical level. So, in the tools described in $[17,18]$ a U-shaped magnetic system is used, which is characterized by significant magnetic flux scattering. Accordingly, such devices have low lifting forces, despite the use of Nd-Fe-B magnets. Magnetic systems of armor type [19], which have the advantage of minimizing the scattering of the magnetic field and the possibility of transformation of magnetic flux density, significantly increase the power characteristics of the tool. In order to maximize the use of permanent magnet energy in armored magnetic systems, it is important to establish rational geometric relationships between permanent magnets and magnetic cores.

The calculation of the complex criterion confirms the presented theses. In the studied tools [14, 15] high values of the criterion $k_{c}$ (up to 0.6 ) are obtained due to the implementation of the proposed technical decisions. For comparison, $k_{c}$ is determined for systems with ceramic magnets, it varies in the range $0.15-0.22$ [13]. The criterion $k_{c}$ can be calculated for various magnetic lifting tools, for example, magnetic tools with a moving magnetic system [20] or tools for cleaning a wellbore [1].

Thus, the proposed complex criterion makes it possible to assess the efficiency of using the energy of permanent magnets in the force dimension, that is, the perfection of magnetic systems of fishing devices. Together with the results of qualimetric analysis, it is possible to thoroughly assess the technical level of the magnetic tool. The above methods may be used along with existing criteria for determining the technical level.

\section{CONCLUSIONS}

A complex criterion has been developed for assessing the technical level of magnetic fishing tools of any size and design. Due to the ratio of actual and theoretically achievable lifting force values, the proposed criterion, in comparison with existing methods, makes it possible to objectively assess the perfection of fishing magnets. The value $k_{c}$ is calculated for magnetic systems with rare-earth and ceramic permanent magnets, which are in the range of 0.53-0.59 and 0.15-0.22, respectively.
As a result of the qualimetric analysis of the passport parameters of 185 serial models of magnetic fishing tools, mathematical models of the average and modern world technical level are obtained. The obtained results allow to assess the technical level of tools at the stage of development and testing of a prototype, to make reasonable decisions on future competitiveness or the need for changes in the range of production.

Based on the results of theoretical studies, technical decisions are proposed that should be implemented in magnetic fishing tools to ensure a high technical level. It has been established that the use of armor-type magnetic systems based on highly coercive rare-earth magnets with rational geometric dimensions of system elements will make it possible to obtain high parameters of fishing tools with the maximum use of permanent magnet energy.

\section{REFERENCES}

[1] B. Coll, G. Laws, J. Jenpert, M. Sportelli, C. Svoboda, and M. Trimble, "Specialized Tools for Wellbore Debris Recovery," Oilfield Review, vol. 24, no. 4, pp. 4-13, 2012.

[2] E. Johnson, J. Land, M. Lee, and R. Robertson, "Landing the big one - the art of fishing," Oilfield Review, vol. 24, no. 4, pp. 26-35, 2012.

[3] P. Connell and D. Haughton, "Removal of Debris from Deepwater Wellbores Using Vectored Annulus Cleaning Systems Reduces Problems and Saves Rig Time," presented at the SPE Annual Technical Conference and Exhibition, Dallas, 2005. https://doi.org/10.2118/96440-MS.

[4] D. Haughton and P. Connell, "Reliable and Effective Downhole Cleaning System for Debris and Junk Removal," presented at the SPE Asia Pacific Oil and Gas Conference and Exhibition, Adelaide, South Australia, Australia, 2006. https://doi.org/10.2118/101727-MS.

[5] E. A. Akhmadullin, V. I. Balaba, V. S. Zaretsky, and V. Ya. Kershenbaum, "Quantitative assessment of repair work quality in wells," Construction of Oil and Gas Wells on Land and Sea, vol. 8, pp. 14-18, 2012.

[6] J. A. Short, Prevention, Fishing, and Casing Repair. Tulsa: PennWell Publishing, 1995.

[7] A. V. Panevnik, I. F. Kontsur, and D. A. Panevnik, "Determination of operating parameters of near-bit ejector assembly," Oil Industry, vol. 10, pp. 10-20, 2018.

[8] I. R. Minnakhmetov and R. N. Talipov, "Classification of the tool for well bottom cleaning from metal," Mining Informational and Analytical Bulletin, vol. 2, pp. 389-391, 2012.

[9] J. Douglas, "Fishing techniques for drilling operations," in Proc. AAPG Southwest Section Meeting, 1999, pp. 15-24.

[10] J. DeGeare, The Guide to Oilwell Fishing Operations: Tools, Techniques, and Rules of Thumb, 2nd ed. Oxford: Gulf Professional Publishing, 2014.

[11] R. R. Zeynalov, "Investigation of magnetic drilling systems of magnetic catcher," Construction of Oil and Gas Wells on Land and Sea, vol. 7, pp. 8-10, 2009.

[12] M. T. Kobyljanskij, "Choice and justification of the main functional criteria for assessing the technical level of magnetic fishing tools," Bulletin KuzSTU, vol. 2, pp. 15-18, 2003.

[13] Y. A. Kurnikov, I. F. Koncur, M. T. Kobyljanskij, and L. I. Romanyshyn, Magnetic fishing tools for well cleaning. Lviv, Ukraine: High school Publishing, 1988. 
[14] E. I. Kryzhanovsky, P. N. Raiter, L. I. Romanishin, and T. L. Romanishin, "Experimental research of characteristics of magnetic systems of the fishing tools," Oil Industry, vol. 7, pp. 104-106, 2014.

[15] T. Romanyshyn, A. Dzhus, and L. Romanyshyn, "Design and research of fishing tools with rational parameters of magnetic systems," Eastern-European Journal of Enterprise Technologies, vol. 4, no. 5, pp. 17-22, 2017. doi:10.15587/1729-4061.2017.10882.

[16] A. Goldman, Handbook of Modern Ferromagnetic Materials. Boston, MA: Springer, 1999. https://doi.org/10.1007/978-1-4615-4917-8.

[17] R. A. Gasanov, R. G. Amirov, and Z. E. Eivazova, "Development of new parametric series of magnetic extractors on the basis of highly energetic magnetic catching devices," Oilfield Engineering, vol. 10, pp. 39-41, 2009.

\section{Taras Romanyshyn}

ORCID ID: 0000-0002-0856-1537

Ivano-Frankivsk National Technical University

of Oil and Gas

Institute of Mechanical Engineering

Karpatska St., 15, 76019, Ivano-Frankivsk, Ukraine

e-mail: tarasromanushun@gmail.com

\section{Liubomyr Romanyshyn}

ORCID ID: 0000-0002-4936-4943

Ivano-Frankivsk National Technical University

of Oil and Gas

Institute of Mechanical Engineering

Karpatska St., 15, 76019, Ivano-Frankivsk, Ukraine

e-mail: romanyshynl@gmail.com

\section{Michał Bembenek}

ORCID ID: 0000-0002-7665-8058

AGH University of Science and Technology

Faculty of Mechanical Engineering and Robotics

al. Mickiewicza 30, 30-059 Krakow, Poland

e-mail: bembenek@agh.edu.pl

\section{Ihor Mokhnii}

ORCID ID: 0000-0003-3852-3934

Branch "UGV-SERVICE" JSC “Ukrgasvydobuvannya"

Raisa Kyrychenko St., 11a, 36039 Poltava, Ukraine

e-mail: igor.mokhniy@ugv.com.ua
[18] I. Ya. Shirali and T. M. Tagieva, "Design concept and parametric line of well magnetic devices," Construction of Oil and Gas Wells on Land and Sea, vol. 12, pp. 5-8, 2013.

[19] D. M. Kobyljanskij, T. V. Bogdanova, and M. T. Kobyljanskij, "Analysis and development of the catcher drilling tool with multiply coonnected magnetic drilling systems," in Proc. VI International Conf. New Word in Scince and Practice, vol. 3, 2017, pp. 6-16.

[20] T. Romanyshyn, V. Sheketa, L. Romanyshyn, and M. Buchynskyi, "Study of the efficiency of the large-diameter fishing tools with the moving magnetic systems," New Tends in Production Engineering, vol. 2, no. 1, pp. 206-213, 2019. doi:10.2478/ntpe-2019-0021. 